\title{
SUBNOTIFICAÇÃO DA TUBERCULOSE: APLICAÇÃO DA METODOLOGIA CAPTURA-RECAPTURA
}

\author{
Maria Aparecida Figueredo Rodrigues ${ }^{b}$ \\ Eduardo Luiz Andrade Motac
}

\section{Resumo}

Este estudo teve como objetivo estimar a subnotificação dos casos de tuberculose no estado da Bahia no ano de 2006, utilizando como fontes de dados o Sistema de Informação de Agravos de Notificação (Sinan) e o Sistema de Informação sobre Mortalidade (SIM). Trata-se de um estudo epidemiológico e descritivo de dados secundários das notificações dos casos novos de tuberculose da Bahia diagnosticados em 2006. Para integração entre os bancos de dados, empregou-se o método probabilístico; para estimativa de casos, utilizou-se a técnica captura-recaptura. Os resultados apontaram que a subnotificação pontual, utilizando a metodologia captura-recaptura, foi de 59,4\%; sem considerar a estimativa, constatou-se que 59,6\% dos óbitos por tuberculose captados pelo SIM como causa básica associada ou mencionada não foram notificados ao Sinan. Concluiu-se que foi elevada a subnotificação dos casos de tuberculose no estado da Bahia no ano de 2006.

Palavras-chave: Tuberculose. Subnotificação. Captura-recaptura. Sistema de Informação.

\section{UNDERREPORTING OF TUBERCULOSIS:APPLICATION OF CAPTURE-RECAPTURE}

\section{METHODOLOGY}

\footnotetext{
Abstract

This objective of this research was to estimate the underreporting of tuberculosis cases in Bahia within 2006, using data from the Information System on Diseases of Compulsory Declaration (Sinan) and data from the Mortality Information System (SIM). This was an

a Artigo apresentado ao Programa de Pós-graduação em Saúde Coletiva, Instituto de Saúde Coletiva da Universidade Federal da Bahia, para obtenção do título de Mestre em Saúde Coletiva com área de Concentração em Epidemiologia. Salvador, 2010.

b Núcleo Regional de Saúde Leste, Cruz das Almas. Secretaria de Saúde do Estado da Bahia. Cruz das Almas, Bahia, Brasil. c Instituto de Saúde Coletiva. Universidade Federal da Bahia. Salvador, Bahia, Brasil. Endereço para correspondência: Rua Aloísio Silveira, número 151, Primavera, Cruz das Almas, Bahia, Brasil. CEP:44380-000.E-mail: maria1.rodrigues@yahoo.com.br
} 
Revista Baiana de Saúde Pública epidemiological and descriptive study of secondary data from reports of new cases of tuberculosis in Bahia diagnosed in 2006. The probabilistic method was adopted for the integration between the databases and, for the estimation of cases, the capture-recapture methodology was applied. The results indicated that using the capture-recapture methodology, the underreporting counted $59.4 \%$, disregarding the estimate, it was found out that $59.6 \%$ of tuberculosis deaths captured by SIM as the underlying associated or mentioned cause weren't reported to Sinan. In conclusion, the underreporting of tuberculosis cases in Bahia was elevated in 2006.

Keywords: Tuberculosis. Underreporting. Capture-recapture. Information System.

\section{EL SUBREGISTRO DE LA TUBERCULOSIS: APLICACIÓN DE LA METODOLOGÍA DE CAPTURA-RECAPTURA}

\begin{abstract}
Resumen
Este estudio tuvo como objetivo estimar el subregistro de casos de tuberculosis en el estado de Bahía, en 2006, utilizando como fuentes de datos el Sistema de Información de Enfermedades de Declaración Compulsória (Sinan) y el sistema de información sobre mortalidad (SIM). Este es un estudio epidemiológico y descriptivo de los datos secundarios de las notificaciones de nuevos casos de tuberculosis diagnosticados en 2006. Para la integración de las bases de datos se utilizó el método probabilístico y la técnica de captura-recaptura para la estimación de los casos. Los resultados mostraron que la subnotificación puntual fue el 59,4\%; sin tener en cuenta la estimación, se constató que el 59,6\% de las muertes por tuberculosis captados por el SIM como causa básica asociada o mencionada no se notificaron al Sinan. Se concluyó que fue alto el subregistro de casos de tuberculosis en el estado de Bahía en 2006.

Palabras clave: Tuberculosis. El Subregistro. Captura y Recaptura. Sistema de Información.
\end{abstract}

\section{INTRODUÇÃO}

Estima-se que um terço da população mundial esteja infectada pelo bacilo de Koch. As regiões da África e Ásia são as que apresentam maior risco de adoecimento. Em 2015, seis países foram responsáveis por 60,0\% dos novos casos de tuberculose no mundo: a Índia, China, Nigéria, Paquistão e África do Sul.

O Brasil está entre os 22 países do mundo que concentram $80 \%$ dos casos de tuberculose, ocupando a $20^{\underline{a}}$ posição quanto à carga da doença e a $19 \underline{a}$ no que se refere à 
coinfecção tuberculose-HIV. Em 2006 foram registrados mais de setenta mil casos novos ${ }^{1}$ e 4.823 óbitos $^{2}$. Entre as internações hospitalares, essa doença representa a oitava causa entre as doenças infecciosas no país ${ }^{1}$. Nesse mesmo ano, a Bahia ocupou o terceiro lugar em número absoluto de casos novos de tuberculose, com mais de seis mil casos notificados, e o sexto em incidência no país (44,5/100.000 hab.) ${ }^{1}$. Foi o terceiro estado da região Nordeste com maior taxa de mortalidade (3,1/100.000 hab.), abaixo apenas dos estados de Pernambuco $(4,4 / 100.000 \text { hab.) e Ceará (3,2/100.000 hab. })^{2}$.

Em 2006 ocorreram, no estado, 1.259 internações por todas as formas da tuberculose, com elevada taxa de mortalidade hospitalar (10,5\%), sinalizando para a realização do diagnóstico tardio e/ou com o registro de complicações. O valor gasto pelo Sistema Único de Saúde (SUS) com as internações nesse ano ficou em torno de um milhão e cem mil reais. Contudo, nos últimos anos, verifica-se uma redução significativa das taxas de incidência e mortalidade da doença no Brasil, e a Bahia tem demonstrado essa tendência. De 1995 até 2006 ocorreu, no país, uma queda de 33,4\% na incidência da tuberculose e 34,8\% no coeficiente de mortalidade. Na Bahia essa redução foi, respectivamente, de $34,8 \%$ e $16,2 \%^{3}$.

Diante dessa situação, algumas questões merecem ser analisadas, tais como: Essa tendência é real ou outros fatores, como subdetecção e subnotificação de casos, podem contribuir para a redução na incidência-5 ${ }^{4-5}$ Nesse contexto, cabe à vigilância epidemiológica identificar situações em que os dados de notificação são diferentes da verdadeira incidência da doença ${ }^{6}$.

Para a notificação dos casos de tuberculose, utiliza-se o Sistema de Informações de Agravos de Notificação (Sinan). Este ainda apresenta muitos problemas no preenchimento e na digitação das notificações/investigações, que se refletem na qualidade das informações. Outras fontes de informações, como o Sistema de Informação sobre Mortalidade (SIM), o Sistema de Informações Hospitalares (SIH-SUS) e outros sistemas, podem ser utilizadas para identificar os casos de tuberculose não registrados no Sinan.

O objetivo do presente estudo foi estimar a subnotificação dos casos de tuberculose no estado da Bahia no ano de 2006, utilizando como fontes de dados o Sinan e o SIM.

\section{MÉTODOS}

O cenário do estudo foi o estado da Bahia, maior e mais populoso da região Nordeste, com população estimada de 13.950.125 habitantes para o ano de 2006, distribuída entre 417 municípios.

Trata-se de estudo epidemiológico e descritivo de dados secundários das notificações dos casos novos de tuberculose da Bahia diagnosticados em 2006 provenientes 
Revista Baiana de Saúde Pública do Sinan e de óbitos associados a tuberculose ocorridos no mesmo ano, constantes no SIM e que foram codificados de A15 a A19 de acordo com Classificação Internacional das Doenças (CID-10) em qualquer uma das partes da declaração de óbito. Permaneceu no estudo a totalidade de casos registrados em cada uma das fontes após a preparação dos dados: para os casos registrados no Sinan, foram excluídos os registros repetidos e a mudança de diagnóstico; no SIM, verificaram-se as duplicidades. Os dados foram processados no programa Microsoft Excel, e as análises foram realizadas no Stata 10.

Para a integração entre os dois bancos de dados empregou-se o método probabilístico, por não haver variável identificadora única nos dois bancos, mas campos comuns em ambos, com o objetivo de identificar o quanto é provável que um par de registros refira-se a um mesmo indivíduo. Para o linkage entre os bancos de dados, utilizou-se o Programa Reclink III e as etapas para o relacionamento probabilístico ${ }^{7}$. Buscou-se localizar no Sinan os casos registrados no SIM, comparando-se os nomes, a data de nascimento, nome da mãe, sexo e endereço. Quando houve coincidência no nome, na data de nascimento, no sexo, no nome da mãe, endereço e município de residência foram conferidos. A identificação dos pares foi realizada manualmente em todas as etapas realizadas.

Devido à possibilidade de encontrar óbitos por tuberculose registrados no SIM e já notificados no Sinan em outro período ou outro modo de entrada no sistema de informação (transferência, abandono, recidiva), um novo linkage foi realizado com um segundo banco de dados do Sinan com todos os casos registrados no período de 2002 a 2006, exceto os casos novos de tuberculose registrados em 2006.

Para estimativa de casos, utilizou-se a técnica captura-recaptura ${ }^{89-10}$ e aplicou-se a Fórmula de Chapman ${ }^{10}$. Neste estudo, $\mathrm{N}$ foi o número estimado de casos de tuberculose na Bahia em 2006, R foi o número total de casos registrados no Sinan, S foi o número total de casos registrados no SIM e $m$ foram os prováveis pares ou recapturados (comuns às duas fontes). A estimativa foi apresentada pontualmente e por intervalo de confiança de 95\%, por sexo, faixa etária e forma clínica da tuberculose. Os valores estimados de casos de tuberculose, quando relacionados o Sinan, o SIM e o número de casos registrados no Sinan, possibilitaram verificar-se a subnotificação de casos da doença no estado da Bahia.

$$
\begin{aligned}
& \text { Fórmula de Chapman } \quad \mathrm{N}=[((\mathrm{R}+1)(\mathrm{S}+1)) / \mathrm{m}+1)]-1 \\
& \qquad \begin{array}{l}
\operatorname{Var}(\mathrm{N} \text { estimado })=\frac{(\mathrm{R}+1)(\mathrm{S}+1)(\mathrm{R}-m)(\mathrm{S}-\mathrm{m})}{(m+1)(m+1)(m+2)} \\
\mathrm{IC}(95 \%)=[\mathrm{N} \text { (estimado) } \pm 1,96 \sqrt{\operatorname{var}(\mathrm{N} \text { estimado })]}
\end{array}
\end{aligned}
$$


Para o cálculo das incidências, utilizou-se como numerador o número de casos novos de tuberculose na Bahia diagnosticados em 2006, após tratamento do Sinan (5.879), segundo sexo (masculino e feminino), faixa etária (0 a 4 anos, 5 a 14, 15 a 39 anos, 40 a 59 anos e 60 anos e mais) e forma clínica (pulmonar, extrapulmonar e pulmonar + extrapulmonar) e a população estimada para esse mesmo ano no denominador, multiplicado por 100.000 habitantes. As incidências corrigidas foram recalculadas para os casos estimados no estudo e para os casos registrados apenas no SIM (246), somando-se aos registros do Sinan. Os dados populacionais foram provenientes do Instituto Brasileiro de Geografia e Estatística (IBGE) disponibilizado pelo Departamento de Informática do Sistema Único de Saúde (DATASUS). Para os casos de tuberculose registrados no SIM e não notificados ao Sinan, identificou-se o percentual e os grupos de maior subnotificação, considerando a faixa etária, sexo, forma clínica e município de residência.

A pesquisa foi aprovada pelo Comitê de Ética e Pesquisa do Instituto de Saúde Coletiva da Universidade Federal da Bahia, sob Registro n. 054-09/CEP ISC.

\section{RESULTADOS}

No banco de dados de notificações de tuberculose, no ano de 2006, havia 6.188 registros de casos novos para todas as formas clínicas da doença. Destes, 5,0\% foram excluídos do estudo devido aos registros repetidos e à mudança de diagnóstico, permanecendo 5.879 casos novos. Quanto ao SIM, registrou 440 óbitos por tuberculose como causa básica e 107 como causa associada ou mencionada na declaração de óbito, totalizando 547 óbitos. Destes, foram excluídos 4 registros por falta da variável "Nome do paciente". Não foram detectadas duplicidades.

Após a análise dos registros no Sinan e SIM, do total de 6.735 foram excluídos 4,6\%, permanecendo 6.422 casos elegíveis para aplicação do método captura-recaptura. Na primeira análise, o relacionamento dos casos de tuberculose possibilitou a identificação de 167 pares entre as fontes, 5.712 casos captados somente pelo Sinan e 376 pelo SIM (Figuras 1 e 2). No linkage realizado com o segundo banco de dados do Sinan havia 39.096 registros e o SIM com 376, foram identificados 130 pares, os quais foram excluídos do estudo, por não ser caso novo diagnosticado em 2006 ou por ter sido diagnosticado fora do período estudado, permanecendo, para aplicação do método captura-recaptura, 246 casos de tuberculose para SIM (Figura 1). 
Revista Baiana de Saúde Pública

Figura 1 - Fluxograma do linkage dos casos de tuberculose registrados no Sistema de Informação de Agravos de Notificação (Sinan) e no Sistema de Informação sobre Mortalidade (SIM), Estado da Bahia - 2006

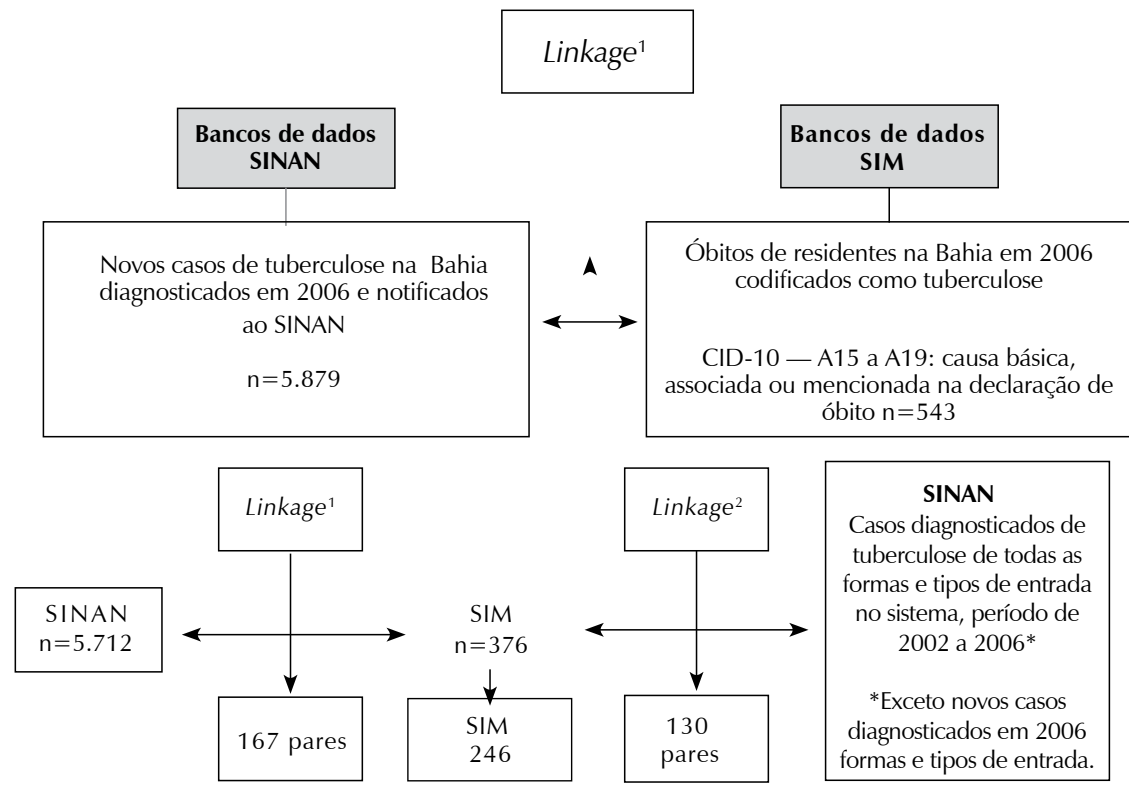

Fonte: Elaboração própria com base em dados do Sinan e do SIM.

${ }^{1}$ Linkage com o primeiro banco de dados do SINAN.

${ }^{2}$ Linkage com o segundo banco de dados do SINAN.

Figura 2 - Número de casos de tuberculose, segundo fontes de captação do Sistema de Informação de Agravos de Notificação (Sinan) e Sistema de Informação sobre Mortalidade (SIM). Estado da Bahia - 2006

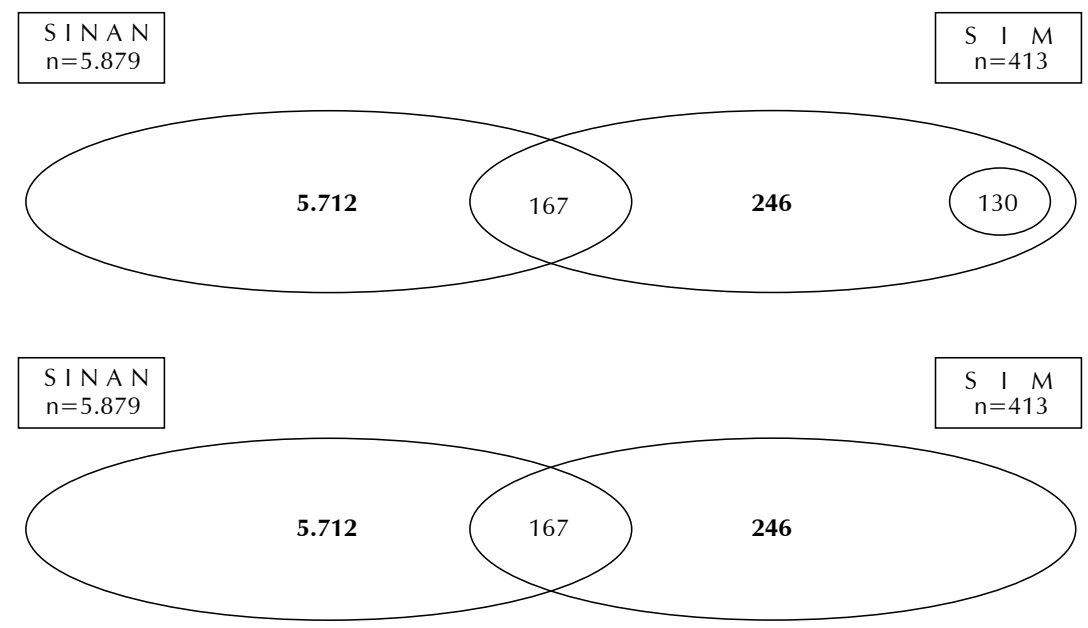

Fonte: Elaboração própria com base em dados do Sinan e SIM. 
Considerando-se cada uma das fontes de dados e com base na captação dos pares entre as duas, utilizando o método probabilístico simples, foram estimados os casos de tuberculose no estado da Bahia em 2006 (Figura 2). Foram encontradas estimativas pontuais de 14.489 casos novos de tuberculose, quando avaliadas as fontes Sinan e SIM, bem como as estimativas por forma clínica, sexo e faixa etária com intervalo de confiança de 95\% (Tabela 1).

Tabela 1 - Estimativa de casos de tuberculose comparando o Sistema de Informação de Agravos de Notificação (Sinan) com o Sistema de Informação sobre Mortalidade (SIM), segundo forma clínica, sexo e faixa etária. Estado da Bahia - 2006

\begin{tabular}{|c|c|c|c|}
\hline \multirow{2}{*}{$\begin{array}{l}\text { Fontes de dados } \\
\text { SINAN x SIM }\end{array}$} & \multicolumn{3}{|c|}{ Estimativas de casos* } \\
\hline & N (estimado) & $\begin{array}{c}\text { Variância } \\
\text { (N estimado) }\end{array}$ & IC (95\%) \\
\hline Estimativa pontual & 14.489 & 717.126 & $12.829-16.123$ \\
\hline \multicolumn{4}{|l|}{ Forma Clínica } \\
\hline Pulmonar & 12.592 & 578.599 & $11.078-14.038$ \\
\hline Extrapulmonar & 1.590 & 102.460 & $976-2.356$ \\
\hline Pulmonar + extrapulmonar & 299 & -33.300 & $-54-604$ \\
\hline \multicolumn{4}{|l|}{ Sexo } \\
\hline Masculino & 8.806 & 398.470 & $7.563-10.049$ \\
\hline Feminino & 5.653 & 311.209 & $4.560-6.746$ \\
\hline \multicolumn{4}{|l|}{ Faixa Etária (anos) } \\
\hline 0 a 4 & 127 & 1.583 & $49-205$ \\
\hline 5 a 14 & 575 & 123.552 & $-114-1.264$ \\
\hline 15 a 39 & 6.437 & 469.274 & $5.094-7.780$ \\
\hline 40 a 59 & 4.189 & 137.796 & $3.462-4.916$ \\
\hline 60 e mais & 2.526 & 69.873 & $2.008-3.044$ \\
\hline
\end{tabular}

Fonte: Elaboração própria, com base em dados do Sinan e do SIM.

* Estimativa realizada pelo método captura-recaptura e aplicação da fórmula de Chapman.

A Tabela 2 apresenta o número de casos e coeficiente de incidência da tuberculose, considerando os registros no Sinan, a soma dos casos notificados ao Sinan com aqueles registrados apenas no SIM e os casos estimados, por forma clínica, sexo e faixa etária. 
Revista Baiana de Saúde Pública
Tabela 2 - Distribuição do número de casos e incidência (100.000 hab.) de tuberculose com base no Sistema de Informação de Agravos de Notificação (Sinan) e do Sistema de Informação de Agravos de Notificação+Sistema de Informação sobre Mortalidade (SIM), estimados segundo forma clínica, sexo e faixa etária. Estado da Bahia - 2006

\begin{tabular}{|c|c|c|c|c|c|c|}
\hline \multirow{3}{*}{ Características } & \multicolumn{6}{|c|}{ Casos de tuberculose } \\
\hline & \multicolumn{2}{|c|}{ Sinan } & \multicolumn{2}{|c|}{ Sinan + SIM } & \multicolumn{2}{|c|}{ Estimados } \\
\hline & $\mathrm{n}^{1}$ & Inc $^{1}$ & $n^{2}$ & $\operatorname{lnc}^{2}$ & $\mathbf{n}^{3}$ & $\ln c^{3}$ \\
\hline \multicolumn{7}{|l|}{ Forma Clínica } \\
\hline Pulmonar & 5.204 & 37,3 & 5.424 & 38,9 & 12.592 & 90,3 \\
\hline Extrapulmonar & 601 & 4,3 & 624 & 4,5 & 1.590 & 11,4 \\
\hline Pulmonar + extrapulmonar & 74 & 0,5 & 77 & 0.5 & 299 & 2,1 \\
\hline Todas as formas & 5.879 & 42,1 & 6.125 & 43,9 & 14.489 & 103,9 \\
\hline \multicolumn{7}{|l|}{ Sexo } \\
\hline Masculino & 3.677 & 53,3 & 3.829 & 55,5 & 8.806 & 127,8 \\
\hline Feminino & 2.202 & 28,6 & 2.296 & 32,5 & 5.653 & 80,0 \\
\hline \multicolumn{7}{|l|}{ Faixa etária (anos) } \\
\hline 0 a 4 & 76 & 5,4 & 78 & 5,5 & 127 & 9,0 \\
\hline 5 a 14 & 143 & 4,7 & 146 & 4,8 & 575 & 18,9 \\
\hline 15 a 39 & 2.881 & 47,4 & 2.939 & 48,3 & 6.437 & 105,9 \\
\hline 40 a 59 & 1.906 & 83,4 & 1.985 & 86,9 & 4.189 & 183,4 \\
\hline 60 e mais & 873 & 76,8 & 977 & 86,4 & 2.526 & 222,4 \\
\hline
\end{tabular}

Fonte: Elaboração própria, com base em dados do Sinan e do SIM.

$\mathrm{n}^{1}=$ casos registrados no SINAN; $\mathrm{n} 2=$ casos registrados no SINAN e casos registrados somente no SIM; $\mathrm{n}^{3}=$ casos estimados; $\mathrm{Inc}^{1}=$ incidência dos casos registrados no SINAN; $I n C^{2}=$ incidência dos registrados no SINAN e casos registrados somente no SIM; Inc $^{3}=$ incidência dos casos estimados.

Houve um incremento de 146,8\% no coeficiente estimado em relação aos dados registrados no Sinan e de $136,7 \%$ após a inclusão ao Sinan dos casos registrados apenas no SIM. A maior incidência foi registrada na forma pulmonar, que apresentou, respectivamente, um aumento de $141,2 \%$ e $131,3 \%$ no coeficiente, quando comparadas as incidências dos casos notificados no Sinan e após a soma dos casos do SIM aos registrados no Sinan. Na extrapulmonar, o incremento foi de $176 \%$ e na pulmonar+extrapulmonar foi de $300 \%$, quando analisada a incidência estimada em relação à incidência para os casos registrados no Sinan.

Comparando-se as incidências por faixa etária, observou-se que os coeficientes mais elevados foram encontrados nos grupos de 40 anos e mais (Tabela 2). Quando analisada a incidência estimada, esta foi maior no grupo com mais de 60 anos, com 222,4/100.000 habitantes. Esse grupo etário também apresentou o maior incremento no coeficiente (12,5\%), 
quando comparada a incidência após a soma dos casos não notificados com aquela obtida com registros do Sinan. No entanto, quando comparada a incidência estimada com o coeficiente dos casos registrados no Sinan, o maior incremento ocorreu na faixa etária de 0 a 14 anos (222,4\%).

Em relação ao sexo, as maiores incidências foram registradas para os homens, com incremento de $138,0 \%$ para a taxa estimada e 4,1\% após a soma dos casos registrados somente no SIM em relação à incidência a calculada com base nos casos notificados no Sinan. Para as mulheres, houve um aumento significativo na incidência estimada, quando comparada à incidência obtida com os casos registrados no Sinan, passando de 28,6 para 80,0/100.000 habitantes, com um incremento de $179,0 \%$ no coeficiente estimado. Após a inclusão dos casos subnotificados, houve um aumento de $13,6 \%$ sobre a incidência calculada com base no Sinan.

A Tabela 3 demonstra que, dos 413 óbitos que tiveram a tuberculose como causa básica, associada ou mencionada, apenas 40,4\% foram informados ao Sinan. Considerando as formas clínicas, essa subnotificação foi maior para a forma pulmonar + extrapulmonar (100\%), seguidas da extrapulmonar (63,8\%) e pulmonar (58,8\%).

Tabela 3 - Óbitos por tuberculose como causa básica, associada ou mencionada registrados no Sistema de Informação sobre Mortalidade (SIM) e número e proporção de óbitos subnotificados no Sistema de Informação de Agravos de Notificação (Sinan), segundo sexo, forma clínica e faixa etária. Estado da Bahia - 2006

\begin{tabular}{|c|c|c|c|}
\hline \multirow{2}{*}{ Características } & \multicolumn{3}{|c|}{ SIM } \\
\hline & Óbitos & Subnotificados & $\%$ \\
\hline \multicolumn{4}{|l|}{ Forma Clínica } \\
\hline Pulmonar & 374 & 220 & 58,8 \\
\hline Extrapulmonar & 36 & 23 & 63,8 \\
\hline Pulmonar + extrapulmonar & 3 & 3 & 100,0 \\
\hline Total & 413 & 246 & 59,6 \\
\hline \multicolumn{4}{|l|}{ Faixa etária (anos) } \\
\hline 0 a 4 & 4 & 2 & 50,0 \\
\hline 5 a 14 & 3 & 3 & 100,0 \\
\hline 15 a 39 & 104 & 58 & 55,8 \\
\hline 40 a 59 & 144 & 79 & 54,9 \\
\hline 60 e mais & 158 & 104 & 65,8 \\
\hline Total & 413 & 246 & 59,5 \\
\hline \multicolumn{4}{|l|}{ Sexo } \\
\hline Masculino & 260 & 152 & 58,5 \\
\hline Feminino & 153 & 94 & 61,5 \\
\hline Total & 413 & 246 & 59,5 \\
\hline
\end{tabular}

Fonte: Elaboração própria, com base em dados do Sinan e do SIM. 
Revista Baiana de Saúde Pública
Considerando os óbitos não informados ao Sinan, a TB pulmonar (CID-10: A162) e a TB respiratória não especificada (CID-10: A169), ambas sem menção de resultado de baciloscopia ou cultura, representaram $76,0 \%$, sendo que em $69,8 \%$ (172), a localização foi pulmonar. A TB pulmonar (CID-10: A153) confirmada por meio não especificado e a TB respiratória não especificada (CID-10: A159) com confirmação bacteriológica e histológica corresponderam a 13,4\%. Para os óbitos em que a tuberculose estava associada, em 22 (8,9\%) casos, a causa básica foi a síndrome da imunodeficiência adquirida, em 11 (4,5\%) casos foi neoplasia e 16 (6,5\%) casos demais causas. Quanto ao local de ocorrência, 71,5\% foi na unidade hospitalar, seguido de $21,1 \%$ no domicílio e 7,3\% em outros locais.

Entre os óbitos registrados no SIM e não notificados ao Sinan, verificou-se que a subnotificação foi maior para as mulheres (Tabela 3). No entanto, analisando apenas os óbitos subnotificados ao Sinan, encontrou-se maior proporção para os homens, correspondendo a 61,8\%. Na Tabela 4, entre os óbitos que não foram notificados no Sinan, a forma pulmonar apresentou maior proporção, correspondendo a 89,4\%.

Tabela 4 - Número e proporção dos casos de tuberculose subnotificados no Sistema de Informação de Agravos de Notificação (Sinan), segundo sexo, forma clínica e faixa etária. Estado da Bahia - 2006

\begin{tabular}{|c|c|c|}
\hline \multirow{2}{*}{ Características } & \multicolumn{2}{|c|}{ Óbitos subnotificados } \\
\hline & $\mathbf{N}$ & $\%$ \\
\hline \multicolumn{3}{|l|}{ Forma Clínica } \\
\hline Pulmonar & 220 & 89,4 \\
\hline Extrapulmonar & 23 & 9,4 \\
\hline Pulmonar + extrapulmonar & 3 & 0,8 \\
\hline Total & 246 & 100,0 \\
\hline \multicolumn{3}{|l|}{ Faixa etária (anos) } \\
\hline 0 a 4 & 2 & 0,8 \\
\hline 5 a 14 & 3 & 1,2 \\
\hline 15 a 39 & 58 & 23,6 \\
\hline 40 a 59 & 79 & 32,1 \\
\hline 60 e mais & 104 & 42,3 \\
\hline Total & 246 & 100.0 \\
\hline \multicolumn{3}{|l|}{ Sexo } \\
\hline Masculino & 152 & 61,8 \\
\hline Feminino & 94 & 38,2 \\
\hline Total & 246 & 100,0 \\
\hline
\end{tabular}


Dentre o total de óbitos, todos os grupos etários apresentaram elevada subnotificação (Tabela 3). Considerando os óbitos não informados ao Sinan, a subnotificação dos casos de tuberculose ocorreu em todos os grupos etários, entretanto os maiores de 60 anos apresentaram as maiores proporções, seguidos da faixa etária de 40 a 59 anos. Entre os menores de 15 anos, o percentual de subnotificação foi de $2 \%$ (Tabela 4).

Para os óbitos por tuberculose notificados ao Sinan, a média da idade foi de 51,9 anos e a mediana de 50 anos (DP $\pm 19,5$ ). Entre os não notificados a média de idade foi de 54,6 e a mediana de 55 anos (DP $\pm 20,8$ ). A média da idade dos casos não notificados foi maior que a dos notificados. Comparando-se o sexo em relação à idade, $50 \%$ dos casos subnotificados estão acima 55 anos para os homens e 54 para as mulheres.

Quanto aos municípios da Bahia, 118 apresentaram pelo menos um caso de tuberculose registrado no SIM e não informado ao Sinan. Dentre estes, 30 municípios são responsáveis por $63,4 \%$ dos casos não notificados e Salvador foi o município que mais contribuiu para a subnotificação, com 30,1\% (Figura 3).

Figura 3 - Distribuição dos casos de tuberculose registrados no Sistema de Informação sobre Mortalidade (SIM) e subnotificados ao Sistema de Informação de Agravos de Notificação (Sinan). Estado da Bahia - 2006
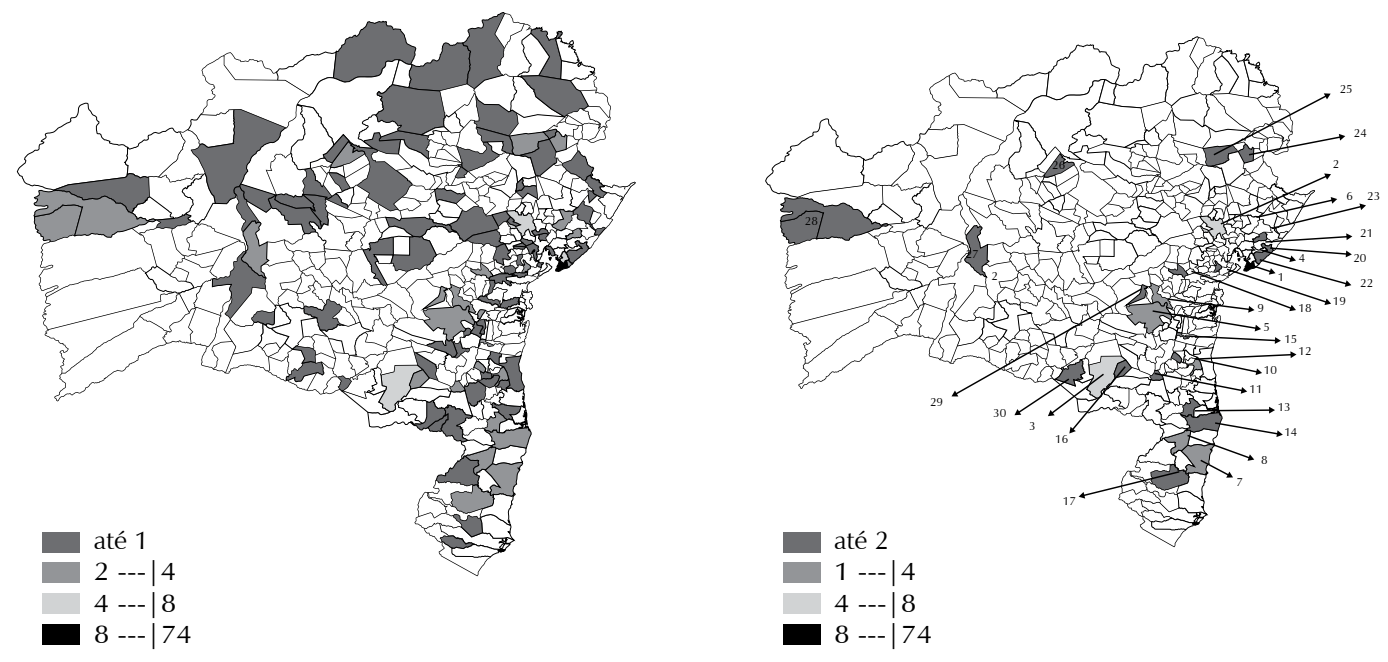

Municípios que tiveram casos de tuberculose registrados no Sistema de Informação sobre Mortalidade e subnotificados ao Sistema de Municípios responsáveis por mais de $60 \%$ subnotificações: 1. Salvador; 2. Feira de Santana; 3. Vitória da Conquista;

4. Simões Filho; 5. Jequié; 6. Alagoinhas; 7. Porto Seguro;

8. Eunápolis; 9. Jaguaquara; 10. Itabuna; 11. Itororó;

12. Almadina; 13. Mascote; 14. Belmonte; 15. Gongogi;

16. Barra do Choça; 17. Itamaraju; 18. Amargosa; 19. Nazaré;

20. Camaçari; 21. Dias D'Ávila; 22. Lauro de Freitas;

23. Pojuca; 24. Ribeira do Pombal; 25. Quijingue;

26. São Gabriel; 27. Paratinga; 28. Barreiras;

29. Lajedo do Tabocal; 30. Tremedal.

Fonte: Elaboração própria, com base em dados do Sinan e do SIM. 
Revista Baiana de Saúde Pública

\section{DISCUSSÃO}

O presente estudo identificou alto percentual de subnotificação de casos de tuberculose e deve servir de alerta para a vigilância epidemiológica da doença. Considerando a estimativa obtida com a utilização da metodologia captura-recaptura, a subnotificação pontual dos casos de tuberculose na Bahia foi de 59,4\%. Aplicando-se a metodologia captura-recaptura e diferentes sistemas de informação, alguns estudos ${ }^{10-11}$ apontaram elevada subnotificação para diversas doenças de notificação compulsória, semelhante ao encontrado nesta pesquisa. Estudo realizado no Brasil identificou que a subnotificação para leishmaniose visceral foi de 42,2\% e 45,0\%, respectivamente, quando comparado Sinan com SIH e SIM ${ }^{10}$. Pesquisa realizada em alguns municípios brasileiros encontrou que a subnotificação dos casos de AIDS variou de $24 \%$ a $65 \%{ }^{11}$.

Sem considerar nenhuma estimativa, constatou-se que 59,5\% dos óbitos por tuberculose captados pelo SIM como causa básica, associada ou mencionada não foram notificados ao Sinan. Essa elevada subnotificação dos casos de tuberculose no Sinan não difere do encontrado em outros estudos de avaliação dos registros da doença quando comparados Sinan e SIM. Pesquisa realizada no Rio de Janeiro verificou que 58,3\% dos casos de tuberculose que evoluíram para óbito não constavam no Sinan ${ }^{12}$. Em outro estudo, realizado em Fortaleza, esse percentual foi de $66,0 \%{ }^{5}$. Para avaliar a subnotificação e resgatar os casos não notificados em Fortaleza, foram comparados os casos informados ao Sinan com os registrados pelas unidades de saúde nos livros de acompanhamento dos pacientes de tuberculose, no período de 2000 a 2002 e encontrou-se subnotificação de $18,5 \%{ }^{4}$.

Dos 30 municípios baianos que contribuíram com mais de $60 \%$ de subnotificação de casos de tuberculose em 2006, oito são prioritários para o Programa Nacional de Controle da Tuberculose. O município de Salvador apresentou a maior proporção de casos subnotificados e respondeu por $36,1 \%$ dos casos novos de tuberculose notificados no estado. Estudo identificou que, embora todos os municípios brasileiros enviem notificações ao Sinan, a entrada direta de dados informatizados ocorre em cerca de $70,0 \%{ }^{13}$. Essa dificuldade em registrar os casos de tuberculose no Sinan foi demonstrada em estudo que identificou deficiência na capacidade de um em cada quatro municípios de captar casos novos de TB ou de registrar adequadamente os dados de notificação e acompanhamento, o que pode indicar a existência de importantes áreas de subnotificação da TB e precário funcionamento do programa de controle da doença ${ }^{6}$.

Considerando o alto percentual de subnotificação dos casos que evoluíram para óbito e que 89,4\% foram da forma pulmonar, pode-se perceber que o diagnóstico está ocorrendo 
tardiamente e quando o paciente apresenta complicações, portanto, com necessidade de internação hospitalar. A falta de notificação dos casos de tuberculose que evoluíram para o óbito pode sugerir, segundo estudo realizado, que o diagnóstico ocorreu na forma mais avançada da doença, quando foi necessária a internação para o diagnóstico e tratamento ${ }^{5}$. O problema torna-se mais grave quando se considera que 90,5\% dos óbitos tinham a forma pulmonar da doença, teoricamente mais fácil de ser diagnosticada.

Por outro lado, chama atenção a elevada proporção de óbitos sem menção de confirmação por resultado de exames, conforme codificação analisada e considerando a Classificação Internacional das Doenças (CID-10), quando comparada ao volume das internações hospitalares e à média de permanência: apenas 13,4\% tiveram diagnóstico confirmado, embora $71,5 \%$ dos óbitos tivessem ocorrido em hospitais. Essa situação não permite saber se os exames diagnósticos não estão sendo realizados ou se a declaração de óbito não está sendo preenchida ou codificada adequadamente.

Dos óbitos subnotificados, 8,9\% tinha a comorbidade TB-Aids. O Programa Nacional de Controle da Tuberculose ${ }^{14}$ e o Programa Nacional de DST/AIDS tornaram obrigatória a oferta e a realização do teste HIV para os casos com diagnóstico de tuberculose, favorecendo ao diagnóstico precoce, tratamento adequado e consequentemente a redução dos óbitos pela comorbidade. De acordo com os dados do Sinan, em 2006, no Brasil, a proporção de casos novos de tuberculose com resultados de HIV era de 43,3\%, sendo 8,5\% positivos. Na Bahia, nesse mesmo ano, apenas $16,7 \%$ dos casos notificados no Sinan tinham sua condição sorológica conhecida. Destes, 3,0\% eram HIV positivos. Esses resultados são preocupantes, pois se perde a oportunidade de identificar a real situação da comorbidade TB-Aids e fornecer subsídios para implementação das ações de vigilância da coinfecção.

Houve diferenças na subnotificação, quando comparados o sexo, a forma clínica e a faixa etária. Embora as médias de idades fossem discretas para os sexos, apresenta-se maior quando comparados os casos não notificados com os notificados ao Sinan.

Os maiores de 60 anos aparecem como importante grupo em relação à subnotificação entre os óbitos. É possível que o diagnóstico nesse grupo esteja ocorrendo tardiamente, seja pela dificuldade de reconhecimento do quadro clínico, que muitas vezes é confundido com as alterações próprias do envelhecimento ou não é referido de forma adequada pelo paciente, seja por demora em procurar o serviço, seja pela dificuldade de acesso aos serviços de saúde. Portanto, ao ser diagnosticada na forma mais avançada da doença tem levado ao óbito ${ }^{15}$. Provavelmente, os óbitos com diagnóstico de tuberculose mais tardio são os menos notificados ao Sinan. 
Revista Baiana de Saúde Pública
Em relação ao acesso aos serviços de saúde, deve-se enfatizar o papel da Atenção Básica para a detecção precoce dos casos de tuberculose e demais ações para controle da doença. No entanto, em alguns municípios do Brasil, demonstrou-se que a descentralização das ações de tuberculose para a Saúde da Família ou ambulatórios tradicionais não apresentou desempenho satisfatório para o acesso ao diagnóstico ${ }^{16}$. Essa situação pode ser reflexo das dificuldades da maioria dos municípios em organizar a atenção básica.

Utilizando o método probabilístico simples, foi possível calcular a estimativa pontual dos casos de tuberculose para a Bahia em 2006, a qual superou as estimativas realizadas pelos métodos recomendados pelo Ministério da Saúde ${ }^{17}$. Pelo método de incremento de descoberta de casos, foram estimados 7.337 casos para 2006 e 10.003 utilizando a estimativa do número de casos de tuberculose com base nos sintomáticos respiratórios (SR). Para aplicação mais precisa desses métodos, seria necessária uma boa organização dos serviços de saúde para aumentar a capacidade de diagnóstico e tratamento da tuberculose.

A distribuição etária registrou incidências mais elevadas da tuberculose para as pessoas acima de 40 anos, tanto para incidência calculada com base nos casos registrados no Sinan quanto para as incidências estimadas, com predominância entre os homens. Estudos apontam que o risco de adoecer por TB aumenta com a idade para ambos os sexos, mas é mais evidente para o sexo masculino ${ }^{18-19}$. Outros trabalhos têm demonstrado essa tendência ${ }^{20-21}$, porém as razões que justifiquem a maior prevalência e incidência da doença no sexo masculino não são ainda bem conhecidas.

Alguns estudos realizados pela OMS $22-23$ apontam que as diferenças nas incidências entre os sexos podem refletir diferenças biológicas (diferenças de sexo), na epidemiologia da tuberculose, nos papéis sociais de homens e mulheres (diferenças de gênero) que influenciam o risco de exposição e/ou diferenças de gênero no acesso aos cuidados. A interação entre os fatores biológicos, socioculturais e as diferenças de gênero deve ser considerada.

Em relação às questões biológicas, as pesquisas têm relatado que as diferenças nas taxas de prevalência e incidência entre os sexos começam a aparecer entre 10 e 16 anos de idade, e continuam a ser maiores para homens do que para as mulheres ${ }^{22-23}$. Outros resultados apresentados pela $\mathrm{OMS}^{24}$ sugerem que, antes da adolescência, há pouca diferença na incidência e prevalência da tuberculose entre os sexos e com as alterações biológicas e as mudanças sociais associadas com esta fase da vida, as diferenças entre ambos aparecem de forma mais acentuada, e os homens começam a superar as mulheres em relação às taxas da doença.

Considerando o viés sexo e imunidade, estudo apontou que, em geral, as mulheres têm a imunidade sistêmica superior, em comparação aos homens. Este resultado 
leva à noção de que esse aumento na função imune confere maior susceptibilidade à autoimunidade, portanto elas são mais propensas a doenças autoimunes, a exemplo da esclerose múltipla, artrite reumatoide, lúpus eritematoso sistêmico, Hashimoto e outras mais prevalentes em mulheres ${ }^{25}$. Os hormônios esteroides sexuais, estrógeno e testosterona, poderiam modular diretamente a função das células envolvidas na resposta imune, mas essa resposta, nas mulheres, varia em diferentes estados hormonais. Além desses fatores, elas têm maior concentração de soro Ig, número de células T CD4 +, e relação CD4/CD8 no sangue; maior produção da citocina em resposta às infecções, maior resposta humoral e das células T a numerosos antígenos, além de maior resistência a infecções. Considerando todos esses mecanismos envolvidos à resposta imunológica, é possível que as mulheres sejam menos propensas a tuberculose.

As diferenças no comportamento de gênero podem contribuir para maior risco da infecção e a progressão da tuberculose ativa, devido a alterações no sistema imune, como o uso de fumo, álcool, a migração e, em algumas situações, a prisão ${ }^{22,24}$. Estudo realizado no Sul da Índia apontou que o risco de progressão da infecção para a tuberculose pulmonar é 8,6\% entre os homens e 3,1\% entre as mulheres. Na população de estudo, praticamente todos os alcoólicos e os fumantes eram do sexo masculino. Após a exclusão dos fumantes e alcoólatras, a relação masculino/feminino reduziu consideravelmente de $2,7-1,2^{22}$.

Nesta pesquisa, chama atenção o significativo aumento na incidência estimada em relação à obtida com os casos registrados no Sinan para o sexo feminino, o que pode indicar a não detecção ou a subnotificação de casos nesse grupo. As barreiras culturais e de acesso relacionados com os serviços de saúde dificultam o diagnóstico oportuno da doença nas mulheres. Outras barreiras sociais para o diagnóstico incluem papéis como os cuidados de outras pessoas, o que dificulta o acesso ao diagnóstico, falta de sensibilidade dos serviços de saúde para as necessidades específicas de gênero, além do estigma e da discriminação das mulheres portadoras da tuberculose ${ }^{26}$.

A alta incidência da tuberculose em maiores de 60 anos pode ser explicada com base em alguns fatores. Um deles seria o crescimento da população idosa, considerando que o aumento da expectativa de vida aumentaria o risco de adoecer por tuberculose, uma vez que a coorte de idosos que foram expostos à doença durante a infância e juventude, quando a prevalência era alta e os esquemas terapêuticos pouco eficazes, mantém a infecção latente. A imunodeficiência devido a uma queda da imunidade celular própria da idade, a sobreposição de doenças crônicas, o uso de drogas imunossupressoras, desnutrição, alcoolismo, tabagismo e 
Revista Baiana de Saúde Pública situação socioeconômica precária, entre outros, devem contribuir para a reativação da infecção latente ${ }^{15,18-19,27-28}$.

A menor ocorrência da doença foi encontrada no grupo menor de 15 anos, embora houvesse um aumento importante para a incidência estimada (15,8/100.000 hab.) e para a proporção de casos estimados para esse grupo (5,0\%), demonstrando, assim, que muitos casos não estão sendo diagnosticados ou notificados. Há dificuldades em diagnosticar os casos nessa faixa etária. Inicia-se o tratamento por meio dos testes terapêuticos sem confirmação diagnóstica, o que, portanto, contribui para a notificação ${ }^{19}$.

\section{CONSIDERAÇÕES FINAIS}

O relacionamento dos bancos de dados possibilitou a identificação de aspectos importantes referentes à notificação dos casos de tuberculose no Sinan. Com a utilização do método captura-recaptura foi possível a estimativa dos casos de tuberculose e, com esses resultados, a verificação pontual da incidência, que se mantém elevada. Conclui-se, portanto, que foi elevada a subnotificação dos casos de tuberculose no estado da Bahia no ano de 2006.

Algumas limitações foram encontradas no estudo. Uma delas refere-se à qualidade dos dados dos sistemas de informações. O Sinan apresenta ainda muitos problemas no preenchimento e na digitação das notificações/investigações, o que se reflete na qualidade das informações. Um aspecto a ser considerado nesse sistema refere-se à falta de preenchimento adequado dos campos de entrada e situação de encerramento dos casos de tuberculose, de modo que é difícil verificar se é duplicidade ou duplo registro. Esse sistema dispõe ainda de ferramenta que facilita a identificação de possíveis duplicidades e duplos registros, mas, para que seja utilizada, é fundamental o preenchimento adequado dos campos.

Quanto ao SIM, demonstrou-se que os óbitos registrados por tuberculose como causa básica, associada ou mencionada em $76 \%$ dos casos, não têm confirmação diagnóstica referida. Portanto, esse sistema de informação traz alguns problemas decorrentes do preenchimento incorreto da declaração de óbito, seleção errada da causa básica, número de óbitos sem assistência médica ou causas mal definidas, além da subnotificação dos óbitos pelo sistema de informação sobre mortalidade devido às deficiências de cobertura.

Por se tratar de um estudo que utilizou dados secundários, não foram trabalhados os prontuários para avaliação da concordância para diagnóstico de tuberculose para os dois sistemas de informação. Portanto, uma das limitações da aplicação do método captura-recaptura em estudos epidemiológicos refere-se ao viés de informação, estando a variável diagnóstica, como uma das preocupações, para que as estimativas sejam válidas. 
Este estudo não buscou identificar as possíveis causas da subnotificação, mas demonstrar a sua magnitude e contribuição para a falsa impressão de redução de casos e sua gravidade quando se analisa o Sinan. Evidenciou também a necessidade de implementar a vigilância epidemiológica da tuberculose, a melhoria na qualidade dos registros no Sinan e SIM e a articulação entre as diversas áreas, principalmente no que diz respeito à utilização dos sistemas de informação e outras fontes.

Considerando a necessidade de fortalecimento e organização dos serviços de vigilância da tuberculose, algumas recomendações serão apresentadas a seguir:

É aconselhável que os serviços de vigilância viabilizem rotinas para o monitoramento e a avaliação dos registros dos casos de tuberculose no Sinan, SIM e outros sistemas de informação, a exemplo do Sistema de Informação Hospitalar (SIH), visando reduzir a subnotificação, diminuir as inconsistências no banco de dados e melhorar a qualidade das informações. Os técnicos da vigilância podem realizar cruzamento dos dados dos diversos sistemas para obtenção e análise das informações epidemiológicas. Essas informações são importantes aliadas para subsidiar o planejamento, a organização e avaliação de serviços de saúde, assim como respaldar a formulação e implementação das políticas de saúde.

Espera-se que a metodologia e as análises realizadas possam ser utilizadas na rotina dos serviços de vigilâncias estaduais e municipais, e que os dados dos vários sistemas de informações em saúde sejam utilizados de forma complementar para subsidiar as ações de controle da tuberculose. O uso do linkage probabilístico de registros para integrar os dados contidos nos diversos sistemas de informações deve ser estimulado e aplicado para outros agravos e doenças, subsidiando as ações de vigilância epidemiológica.

É necessária a articulação entre os gestores dos diversos sistemas de informação, para que se estabeleça uma rotina para disponibilização dos dados dos diversos sistemas para atender às necessidades e demandas dos serviços. Por outro lado, para melhorar a confiabilidade e qualidade da informação, é necessário investir no treinamento dos profissionais para o preenchimento dos instrumentos de coleta e a digitação dos dados, utilização das ferramentas de análise e produção da informação.

Quanto ao acesso aos serviços de saúde para detecção precoce de casos e demais ações de controle da doença, é necessária a reorganização da Atenção Básica na maioria dos municípios e também a reestruturação do processo de trabalho das equipes de saúde. As ações básicas, como abordagem dos sintomáticos respiratórios, consolidação do tratamento diretamente observado, realização de exames diagnósticos e acompanhamento, busca ativa e exames de contados, devem ser realizadas efetivamente. 
Revista Baiana de Saúde Pública
Para os óbitos que tiveram a tuberculose como causa básica, associada ou mencionada na declaração de óbito, a vigilância epidemiológica deverá confirmar se já consta no Sinan. Se ainda não foi notificado, recomenda-se a validação do diagnóstico do óbito, a realização das demais ações de vigilância e o controle da doença pela vigilância e atenção básica.

No que diz respeito às fontes notificadoras, é de suma importância a criação e/ou fortalecimento dos núcleos de vigilância epidemiológica hospitalares, já que essas unidades são importantes fontes de notificação de doenças e agravos e terão o papel importante para o desencadeamento das ações de prevenção e controle da tuberculose.

\section{COLABORADORES:}

1. Concepção do projeto, análise e interpretação dos dados: Maria Aparecida Figueredo Rodrigues e Eduardo Luiz Andrade Mota.

2. Redação do artigo e revisão crítica relevante do conteúdo intelectual: Maria Aparecida Figueredo Rodrigues e Eduardo Luiz Andrade Mota.

3. Revisão e/ou aprovação final da versão a ser publicada: Maria Aparecida Figueredo Rodrigues e Eduardo Luiz Andrade Mota.

4. Ser responsável por todos os aspectos do trabalho na garantia da exatidão e integridade de qualquer parte da obra: Maria Aparecida Figueredo Rodrigues e Eduardo Luiz Andrade Mota.

\section{REFERÊNCIAS}

1. Brasil. Ministério da Saúde. Departamento de informática do SUS (DATASUS). TABNET: Serviços. Epidemiológicas e morbidade. Brasília; 2006 [citado 2009 out 8]. Disponível em: http://www2.datasus.gov.br/DATASUS/ index.php?area $=0203 / /$

2. Brasil. Ministério da Saúde. Departamento de informática do SUS (DATASUS). Estatísticas vitais. Brasília; 2006 [citado 2010 mar 14]. Disponível em: http:// tabnet.datasus.gov.br/cgi/tabcgi.exe?sim/cnv/obt10uf.def 3

3. Brasil. Ministério da Saúde. Secretária de Vigilância em Saúde. Informe eletrônico da tuberculose. Bol Epidemiol. 2009 jul [citado 2009 out 10]; Ano 9(2):2-4. Disponível em: http://portal.saude.gov.br/portal/arquivos/pdf/ boletim_tb_julho09.pdf

4. Façanha MC. Tuberculose: subnotificação de casos que evoluíram para o óbito em Fortaleza-CE. Rev bras Epidemiol. 2005;8(1):25-30. 
5. Façanha MC, Guerreiro MFF, Pinheiro AC, Lima JRC, Vale RLS, Teixeira GFD. Resgate de casos subnotificados de tuberculose em Fortaleza-CE 2000-2002. Bol Pneumol Sanit. 2003;11(2):13-6.

6. Brag JU. Vigilância epidemiológica e o sistema de informação da tuberculose no Brasil, 2001-2003. Rev Saúde Pública. 2007;41(Supl 1):77-88.

7. Camargo JR KR, Coeli CM. RecLink III versão 3.1.6.3160: Guia do usuário. Rio de Janeiro; 2007.

8. Coeli CM, Veras RP, Coutinho ESF. Metodologia de captura-recaptura: uma opção para a vigilância das doenças não transmissíveis na população idosa. Cad Saúde Pública [online]. 2000 [citado 2009 jun 5];16(4):1071-82. Disponível em: http://www.scielo.br/scielo.php?script=sci_ arttext\&pid=S0102-311X2000000400025\&lng=pt\&nrm =iso\&tlng=pt

9. Dunn J, Andreoli SB. Método de captura e recaptura: nova metodologia para pesquisas epidemiológicas. Rev Saúde Pública [online]. 1994 [citado 2009 maio 20];28(6):449-53. Disponível em: http://dx.doi.org/10.1590/S003489101994000600009

10. Maia-Elkhoury ANS, Carmo EH, Sousa-Gomes ML, Mota E. Análise dos registros de leishmaniose visceral pelo método de captura-recaptura. Rev Saúde Pública [online]. 2007 [citado 2009 maio 15];41(6):931-7. Disponível em: http://www.scielo.br/scielo.php?script=sci_arttext\&pid=S003489102007000600007\&lng=pt. http://dx.doi.org/10.1590/S003489102007000600007

11. Oliveira MTC, Barreira D, Santos LCO, Latorre MRDO. A subnotificação de casos de aids em municípios brasileiros selecionados: uma aplicação do método de captura-recaptura. Bol Epidemiol AIDST. 2004 jun [citado 2010 jan 4];Ano 1(1):7-11. Disponível em: http://www.aids.gov.br/final/dados/ BOLETIM2.pdf

12. Selig L, Belo M, Cunha AJLA, Teixeira EG, Brito R, Luna AL, et al. Óbitos atribuídos à tuberculose no estado do Rio de Janeiro. J bras pneumol. [online]. 2004 [citado 2010 maio 5];30(4):335-42. Disponível em: http:// dx.doi.org/10.1590/S1806-37132004000400006

13. Bierrenbach AL, Stevens AP, Gomes ABF, Noronha EF, Glatt R, Carvalho $\mathrm{CN}$, et al. Efeito da remoção de notificações repetidas sobre a incidência da tuberculose no Brasil. Rev Saúde Pública. 2007 [citado 2009 maio 8];41(Supl 1):67-76.

14. Brasil. Ministério da Saúde. Secretaria de Vigilância em Saúde. Guia de Vigilância Epidemiológica. 7. ed. Brasília; 2009. 
Revista Baiana de Saúde Pública
15. Cavalcanti ZR, Albuquerque MFPM, Campello ARL, Ximenes R, Montarroyos $U$, Verçosa MKA. Características da tuberculose em idosos no Recife (PE): contribuição para o programa de controle. J bras pneumol. [online]. 2006 [citado 2010 maio 27];32(6):535-43. Disponível em: http://dx.doi. org/10.1590/S1806-37132006000600011

16. Scatena LM, Villa TCS, Ruffino Netto A, Kritski AL, Figueiredo TMRM, Vendramini SHF, et al. Dificuldades de acesso a serviços de saúde para diagnóstico de tuberculose em municípios do Brasil. Rev Saúde Pública. [online]. 2009 [citado 2010 mar 2];43(3):389-97. Disponível em: http:// dx.doi.org/10.1590/S0034-89102009005000022.

17. Brasil. Ministério da Saúde. Fundação Nacional de Saúde. Centro de Referência Prof. Hélio Fraga. Sociedade Brasileira de Pneumologia e Tisiologia. Controle da tuberculose: uma proposta de integração ensino-serviço. 5a ed. Rio de Janeiro; 2002.

18. Coelho DMM, Viana RL, Madeira ACA. Perfil epidemiológico da tuberculose no município de Teresina-PI, no período de 1999 a 2005. Epidemiol serv saúde. 2010 jan-mar;19(1):33-42.

19. Xavier MIM, Bareto ML. Tuberculose na cidade de Salvador, Bahia, Brasil: o perfil na década de 1990. Cad Saúde Pública. 2007;23(2):445-53.

20. Kusano MSE, Sousa STR, Assis MCM. Tendência da morbimortalidade por tuberculose no Distrito Federal - Brasil. Bol Pneumol Sanit. 2002;10(1):55-60.

21. Vendramini SHF, Gazeta CE, Chiaravalotti Netto F, Cury MR, Meirelles EB, Kuyumjian FG, et al. Tuberculose em município de porte médio do Sudeste do Brasil: indicadores de morbidade e mortalidade, de 1985 a 2003. J bras pneumol. [online]. 2005 [citado 20 mar 8];31(3):237-43.

22. World Health Organization. Department of Gender, Women and Health. Gender and tuberculosis. [internet] Ginebra; 2002 [cited 2010 July 10]. Available from: http://apps.who.int/iris/bitstream/10665/68891/1/a85584.pdf

23. World Health Organization. Gender and tuberculosis: Cross-site analysis and implications of a multi country study in Bangladesh, India, Malawi, and Colombia. Social, Economic and Behavioural Research. Report series n. 3 [internet]. Ginebra; 2006. [cited 2010 July 10]. Available from: http://www.who. int/tdr/publications/tdr-research-publications/gender-tb-multicountry-study/en/

24. World Health Organization. Department of Gender, Women and Health. Gender in tuberculosis research [internet]. Ginebra; 2004 Jan. [cited 2010 July 10]. Available from: http://www.who.int/gender-equity-rights/ knowledge/9241592516/en/ 
25. Nalbabian G, Kovatis S. Understanding sex biases in immunity: effects of estrogen on the differentiation and function of antigen-presenting cells. S. Immunol Res. 2005;31(2):91-106.

26. Allotey P, Gyapong M. Gender in tuberculosis research. Int J Tuberc Lung Dis [internet]. 2008 [citado 2010 July 10];12(7):831-36. Available from: http:// www.who.int/tdr/publications/documents/gender-tb-research.pdf

27. Bierrenbach AL, Duarte EC, Gomes ABF, Sousa MFM. Tendência da mortalidade por tuberculose no Brasil, 1980 a 2004. Rev Saúde Pública. 2007;41(supl.1):15-23.

28. Mishima EO, Nogueira PA. Tuberculose no idoso: estado de São Paulo, 1940-1995. Bol Pneumol Sanit [online]. 2001 [citado 2010 jul 20];9(1):5-11.

Disponível em: http://scielo.iec.pa.gov.br/scielo.php?script=sci_ arttext\&pid=S0103-460X2001000100002\&lng=pt.

Recebido: 28.8.2015. Aprovado: 10.10.2015. Publicado: 20.9.2017. 